\title{
Medicinay lienciasbiológicas
}

ANO U

EDITORIAI.

\section{LA AGREMIACION MEDICA}

Problema viejo 3 largamente discutido la sido esse, al de la agremiar rón oblifatoria de tos médícos ecuatoriajos. Desd.s la época de Espejo, el protomédico quiterio, hasta mestros diss, han siden numerosos los intentos de asociar a todos los médicos en wra sola cntidad clasista. Lat realización más efectiva lo constîtutye Lr Federación Médica det Eculdolor Ia mismo que en los taltimos años, frucins af prestigio y actipidad ds sus tirigentes se ha robustecito grandemente $y$ ha lograto el. «ue las instituciones pabticas la reconozcar - we hecho- como la perdaderi representante de ta clase modica del pais.

Con motivo de un moysecto de ley, segrin el cuat todos los médicos det Ecuador se contertirfan es miembros de ta Fedzración Médica, la discusién

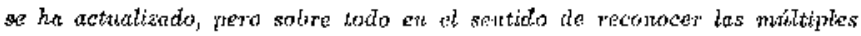
ventajas de que la totalidad de mofesionale!: constituyan una sola arociación clasista. La Fedaración Médica, con ser lin entidad que ha logrado reuntr, en st seno, et maljor ntínero de médios no ha logrado, esprexithente. un. Las dos cindades mís populosas del país, afititt sino a un 50 o $70 \%$ de pro frstionules.

Este hecho limita su Jaorza, su capracidad para impedir pue el médico sea atrapellado - por razonss políticas, jor tjemplo- en sus derechos, en sis estobilidad en el trabajo, en st dingited profesional.

Et Código Sunitario establecs muchos de los derechos det médico, pero en ausencia de un organismo sótido $z$ paderosa que respalde al profesianal. tates derechos ro pasen de ser sine dectamaciones en ct vercia. 
Por ofra parte, ctrmo ha sucedido ya en muchos paises, es necesario que

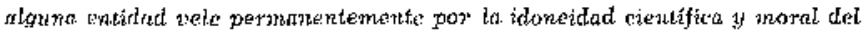
méttirn. El título profesional concerdido por las universidades del país, ciertamente, es el primero e indispenudale paso. Pero las ciencias méclicas no son - ni tejanamente- el limitado acervo de conocimientos que el estadiante adruticre pu las awles waiveryitarias. T'ampoco ba medicine es entotica. Por el coutraria, evolaciona If progrest con und rapites asombrosa. Alguna entidad médica debe responstabilizarse porque sus asociados signa el ritmo de progreso de las comocimientos y del desarrollo de las ciencias; porque el mético, lejos de convertirse en an peligro social por mat ejercicio profesional, siga siendo el salvaguardio de la salud $y$ del bierestar de tos ciudadanos.

Ls necesario, csinzismo, que una entidad médica regule las reknciones emtre profasionales, entre éstos $y$ los pachentes, extre el múdico y las clinicas 2h hospitales, entre los pacientes y estas instituciones. Hay sutiles relaciones que escapan al ámbito de una ley generẩ, como al Código Sanitario.

Todo esto debería estar eft manos de unt Fecheraction Mfédict que agrupe a todos los profesionales y la cual podria contar con sectiones especiales, com funnones esporáficas, coma el Coletrio Médico, pära efactos de la licencia de ejercieio de la medicin, el tribual de traw, parm el fulto sobre niertos conflictos profesiomales, etc.

Por circunstancias de carácter histórico, la Federación Médica ha tobarcado no sólo a médicos sino a los profesionates de tiencias afines cono: Odoptología, Farmacia, Anfermarits, ote. Esta bpoca mede y dele ser superada. La Federación Médic debe csociar sóto a médicos, puts sus problemet.s e intereses profesioneles son, esperíficos, en cambio phtede formarse unu Corfederaciós que asocie en un grande y amplio arganismo a lns varias Federa-

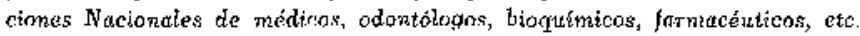

